\title{
Forced Convection Heat Transfer Analysis through Dimpled Surfaces with Different Arrangements
}

\author{
Hasibur Rahman Sardar ${ }^{1}$, Abdul Razak Kaladgi, ${ }^{2}$ * \\ ${ }^{1}$ Department of Electronics \& Communication Engineering, P.A College of Engineering, Karnataka, India \\ ${ }^{2}$ Department of Mechanical Engineering, P.A College of Engineering, Karnataka, India
}

Email address:

hasibpace@gmail.com (H. R. Sardar), abdulkaladgi@gmail.com (A. R. Kaladgi)

\section{To cite this article:}

Hasibur Rahman Sardar, Abdul Razak Kaladgi. Forced Convection Heat Transfer Analysis through Dimpled Surfaces with Different Arrangements. American Journal of Energy Engineering. Vol. 3, No. 3, 2015, pp. 37-45. doi: 10.11648/j.ajee.20150303.12

\begin{abstract}
Dimples play a very important role in heat transfer enhancement of electronic cooling systems, heat exchangers etc. This work mainly deals with the experimental investigation of forced convection heat transfer over circular shaped dimples of different diameters on a flat copper plate under external laminar flow conditions. Experimental measurements on heat transfer characteristics of air (with various inlet flow rates) on a flat plate with dimples were conducted. From the obtained results, it was observed that the heat transfer coefficient and Nusselt number were high for the copper plate in which the diameter of dimples increases centrally in the direction of flow (case c) as compared to the other two cases.
\end{abstract}

Keywords: Forced Convection, Dimples, Heat Transfer, Passive Techniques

\section{Introduction}

There are various heat transfer applications where the use of fluid-to-gas heat exchanger is important. The issues like accurate heat transfer rate analysis, estimations of pressure drops, long-term performance and economic aspect of the equipment make the design of heat exchangers quite complicated. Also higher performance, higher heat transfer rate with minimum pumping power requirements are some of the main challenges of the heat exchanger design. Therefore, improving the heat exchanger efficiency through the enhancement techniques resulting in a considerable reduction in cost is one of main task faced by the engineers [1].Various heat transfer enhancement techniques are developed and used for heat exchanger applications over the past couple of years. Several attempts are also made to reduce the size and cost of the heat exchangers. Among these the passive techniques can be considered important one because of its wide variety of applications like in electronic cooling (heat sinks), process industries, cooling and heating in evaporators, solar air heaters, turbine airfoil cooling etc [2]. The main principle of heat transfer enhancement in passive techniques is the surface modifications such as protrusions, pin fins, and dimples. Among these, the dimples (concavities) can be considered special one as they not only enhance the heat transfer rate but also produce minimum pressure drop penalties [3]. The dimple produces vortex pairs, induces flow separation \& creates reattachment zones to increase the heat transfer. And as they do not protrude into the flow so they contribute less to the foam drag, to produce minimum pressure drop penalties [4]. Another added advantage in dimple manufacture is the removal of material which reduces cost and weight of the equipment.

Kuethe [5] can be considered as the first person to make dimples on flat surfaces to increase the heat transfer rate. According to him the dimples are expected to promote turbulent mixing in the flow, acting as vortex generator \& hence increase the heat transfer rate. Afanasyev et al [6] carried an experimental to study the heat transfer characteristics of flow over a flat plate having spherical dimples and reported an increment of $30-40 \%$ in the heat transfer rate with a minimum pressure drop. Chyu et al [7] conducted an experiment to study local heat transfer coefficient distribution in a channel having dimples of spherical \& tear drop type. They observed a considerable increase in the distribution of local heat transfer coefficient everywhere on these dimple surfaces as compared to flat surface. Mahmood et al [8], experimentally investigated the effect of dimples on heat transfer augmentation. They used the flow visualization techniques and concluded that the periodic nature of shedding off of vortices is the main cause of enhancement of heat transfer and is much more pronounced at the downstream rims of the dimples. Xie et al 
[9] numerically investigated the heat transfer and fluid flow characteristics of teardrop dimple along with teardrop protrusion having different eccentricities. They used the K-E model to capture the turbulence effects. They concluded that the heat transfer enhancement along with energy savings are more in teardrop dimples as compared to flat surfaces.Farhad sangtarash \& hosseinshokuhmand [10] conducted experimental \& numerical investigation on inline \& staggered arrangement of dimples on multilouvered fins to study the heat transfer and pressure drop characteristics of air through these multilouvered fin banks at varying Reynolds number. They concluded that the augumentation of heat transfer was more in staggered arrangements as compared to online arrangement.

From the literature above, it is abundantly clear that dimples or vortex generators and the vortex heat transfer enhancement (VHTE) techniques have a high potential to increase the heat transfer rate along with the production of lower pressure drop penalties. The other advantages are:

a. Fouling rate reduction b. Cost reduction c. weight reduction etc [11], however, much of the research work either experimental or numerical is on spherical dimples of uniform diameter $[7,12]$. It is also seen that most of the research is confined to flow in the channel i.e. Internal flow, with a very few studies on external flow [12]. So the main focus of this experimental work is to study the effect of circular dimples of various diameters under external laminar flow conditions

\section{Experimental Setup}

The prime objective of the present work was to study experimentally the heat transfer enhancement through dimple surfaces of different diameters on a flat plate using force convection technique. For this to be possible we required a forced convection setup which was fabricated as required. The fabricated setup is shown below.

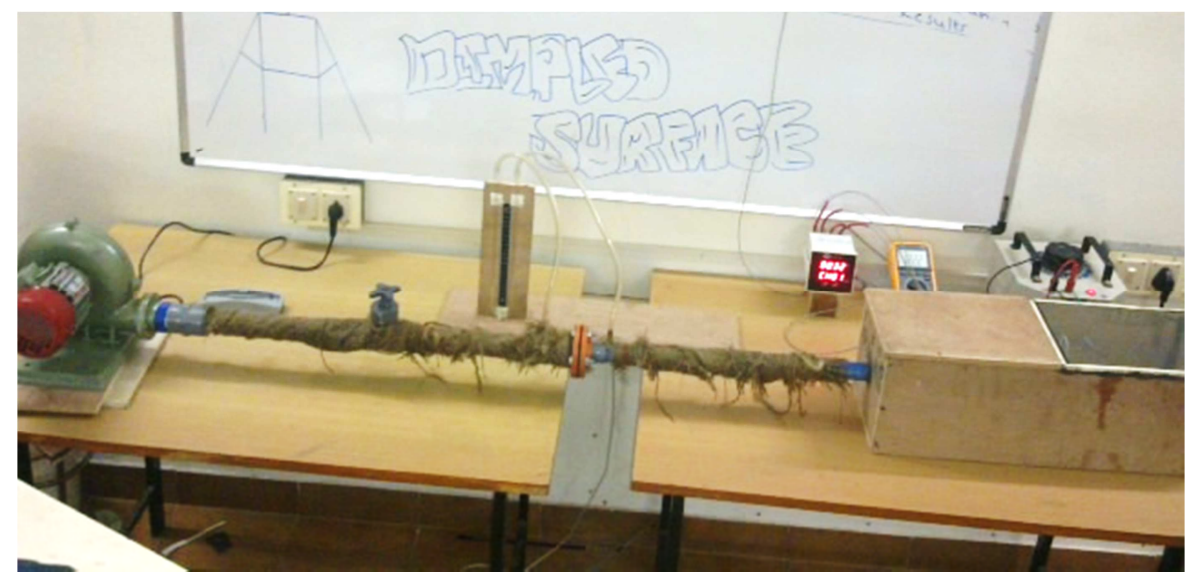

Figure 1. Experimental setup.

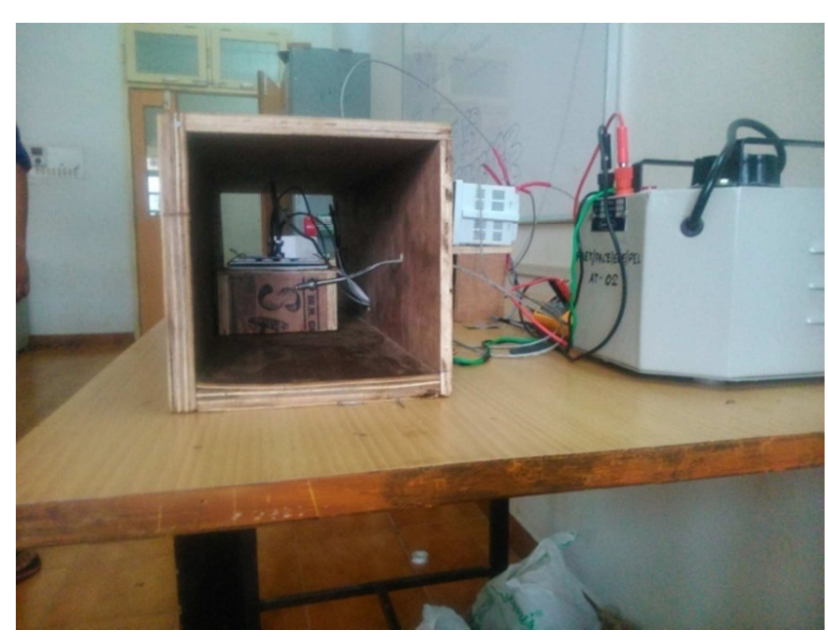

Figure 2. Schematic representation.

The main components of the test apparatus are a test plates of dimensions $100 \times 100 \times 2 \mathrm{~mm}$, a calibrated orifice flow meter, Strip plate heater with capacity of 100 watts, Dimmer stat, Digital temperature, voltmeter, and ammeter with J type thermocouple, a gate valve, and a centrifugal blower. In this work Strip plate heater was fabricated to provide heat input to the test surface. The provision was made to fix the heater at the base of the each test plate in a rectangular channel connect to the blower through a orifice plate with pipe at inlet and to the atmosphere at outlet. U-TUBE manometer was connected across the orifice plate to indicate the pressure difference in terms of centimeters of water column difference. A PVC pipe was used to connect the blower outlet to the rectangular duct. Next to the blower outlet, flow regulating valve was connected to the pipe to regulate the air flow.

Table 1. Components and Specifications.

\begin{tabular}{ll}
\hline Components & Specification \\
\hline Test plate & $10 \times 10 \times 2 \mathrm{~cm}$ copper plates \\
Blower & $110 \mathrm{~W}, 0.4 \mathrm{BHP}, 280 \mathrm{rpm}$ \\
Heater & $100 \mathrm{~W}, 4$ "x4" \\
Dimmer stat & $6 \mathrm{~A}, 230 \mathrm{~V}$ \\
Digital Temperature Indicator & 6 channel, $1200^{\circ} \mathrm{C}, 230 \mathrm{~V}$ \\
Orifice plate & $12 \mathrm{~mm}$ dia. \\
Manometer & "U-tube" glass manometer \\
Casing & A wooden casing of size of 8 "x 8 " and \\
Thermocouple & 2feet long. \\
Digital Multi-meter & K-Type, $300^{\circ} \mathrm{C}, 1 \mathrm{~m}$ long. \\
\hline
\end{tabular}




\section{Results and Discussion}

Experiments were conducted on copper test plates with circular dimples of different diameters. The dimples were arranged in a staggered fashion with different arrangements like:

Case a. Gradual Increase in the diameter of dimples in the left \& right columns of the plate in the direction flow.

Case b. Gradual Decrease in the diameter of dimples in the left $\&$ right columns of the plate in the directionflow (reverse case).
Case c. centrallyincreasing the diameter of dimples in the direction flow\& maintaining the left \& right columnwith constant diameter dimples.

The data obtained were used to find heat transfer parameters like Nusselt number, heat transfer coefficient, and heat transfer rate. And the experimental findings have been plotted in the form of graphs, mainly

- Nusselt number(Nu) vs Reynolds number(Re)

- Heat transfer coefficient(h) vs Reynolds number(Re)

- Heat transfer rate Q vs Reynolds number(Re)

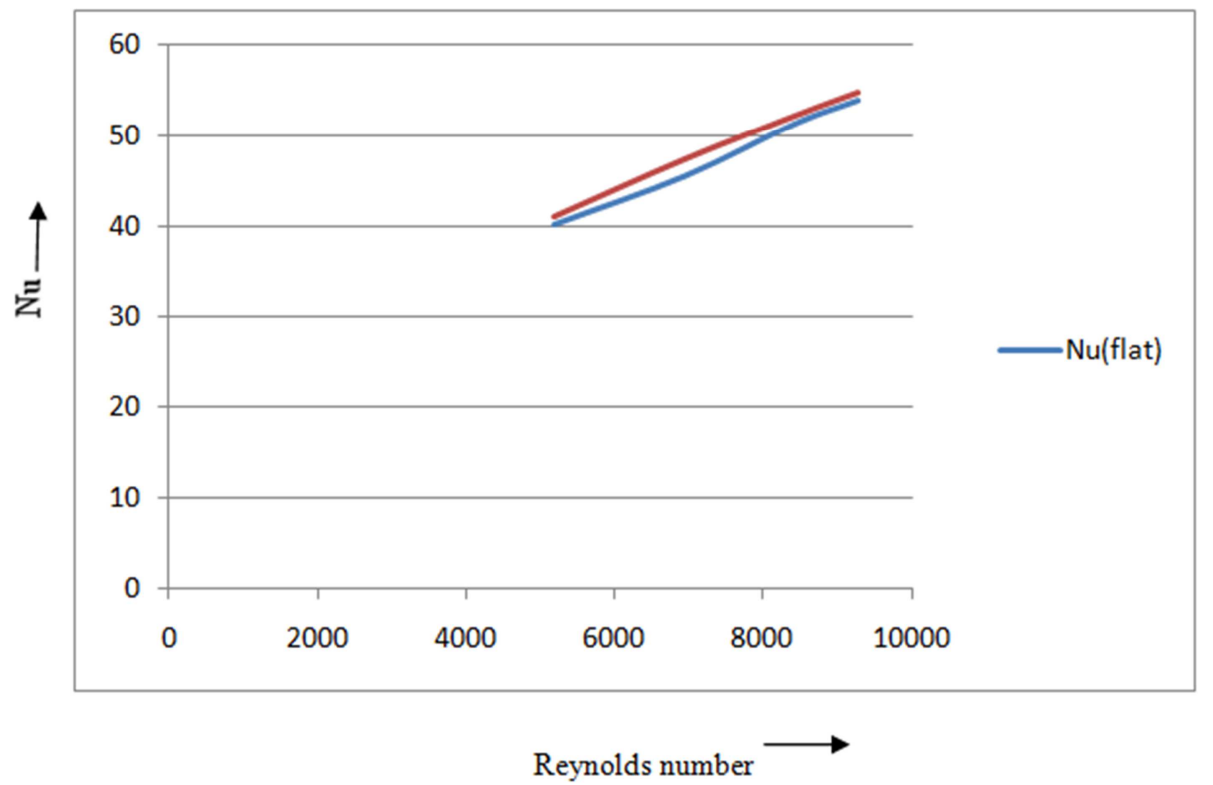

Figure 3. Variation of Nusselt number with Reynolds number(case.a).

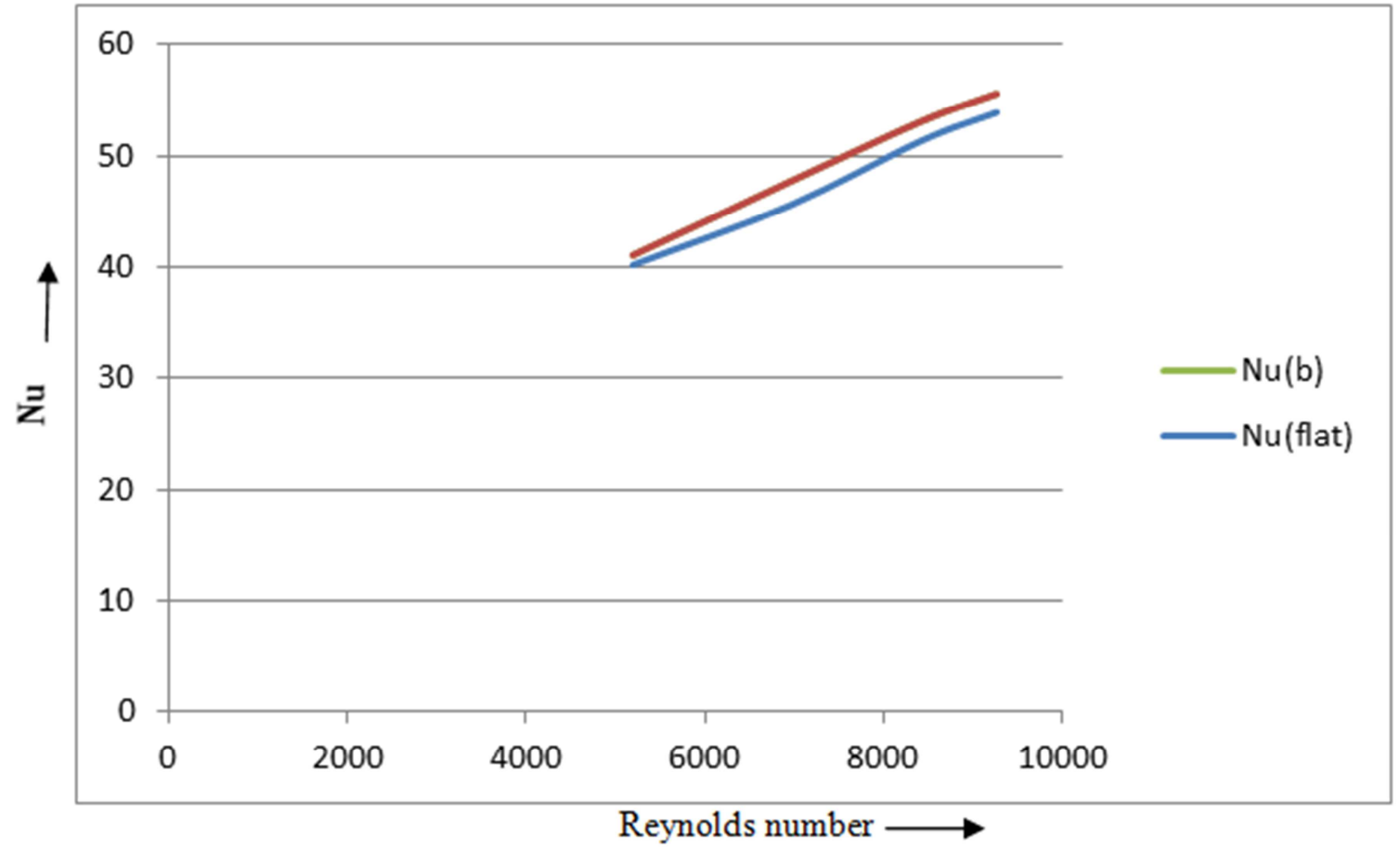

Figure 4. Variation of Nusselt number with Reynolds number(case.b.). 


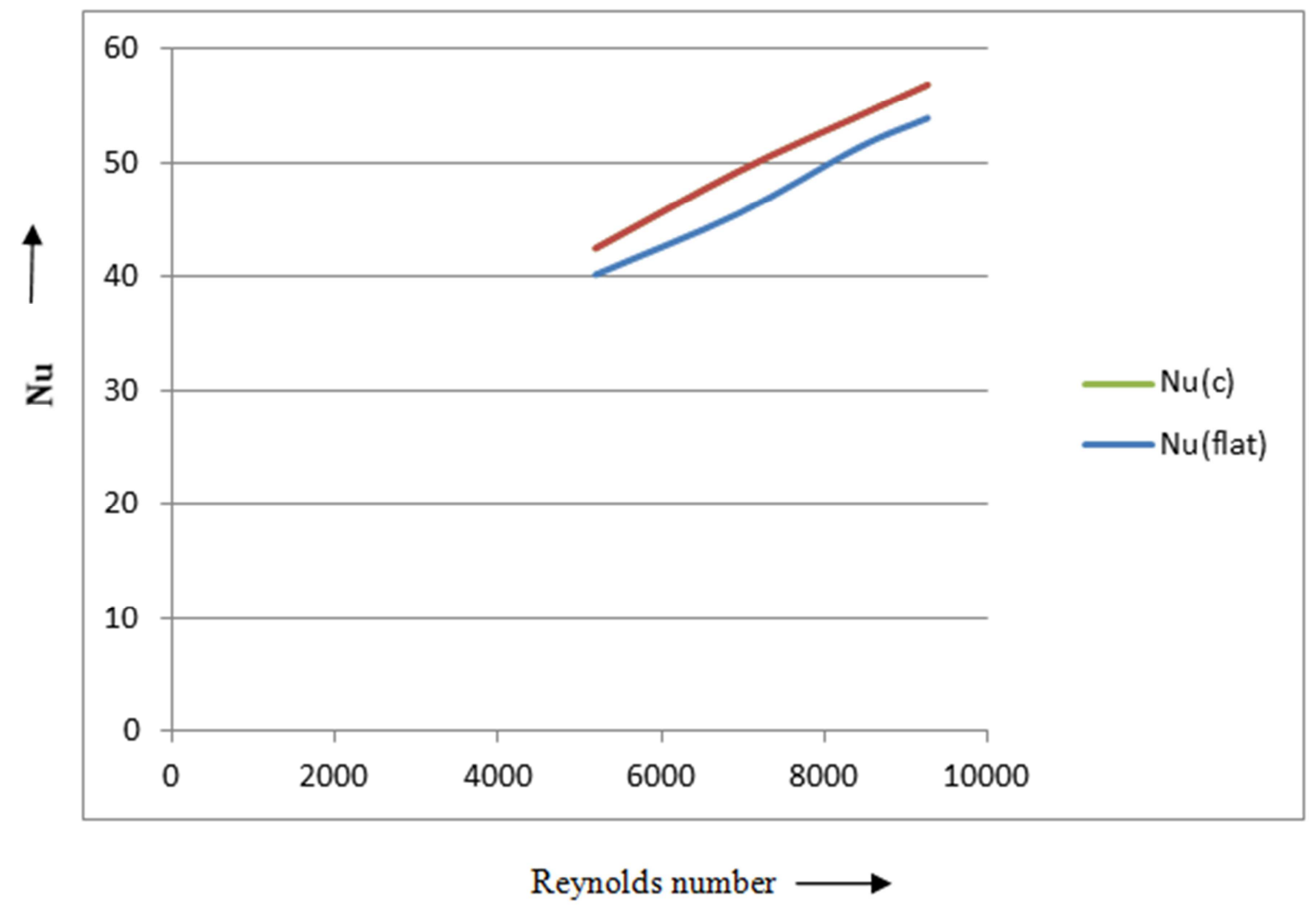

Figure 5. Variation of Nusselt number with Reynolds number(case.c.).

Figure 3, 4, 5 shows variation of Nusselt number ' $\mathrm{Nu}$ ' with Reynolds number for the three cases considered. It is obvious that the ' $\mathrm{Nu}$ ' increases as Reynolds number increases due to direct flow impingement on the downstream boundary and strengthened flow mixing by vortices at the downstream $[3,13]$. The formation of vortex pairs which are periodically shed off from the dimples, a large up wash regions with somefluids coming out from the central regions of the dimples, from vortex pairs $\&$ near dimple diagonals are the main causes of enhancement of Nusselt number \& is more pronounced near the downstream rims of the dimples [8].It can also be seen that the variation in the Nusselt number is gradual with Reynolds number as expected [14, 15].

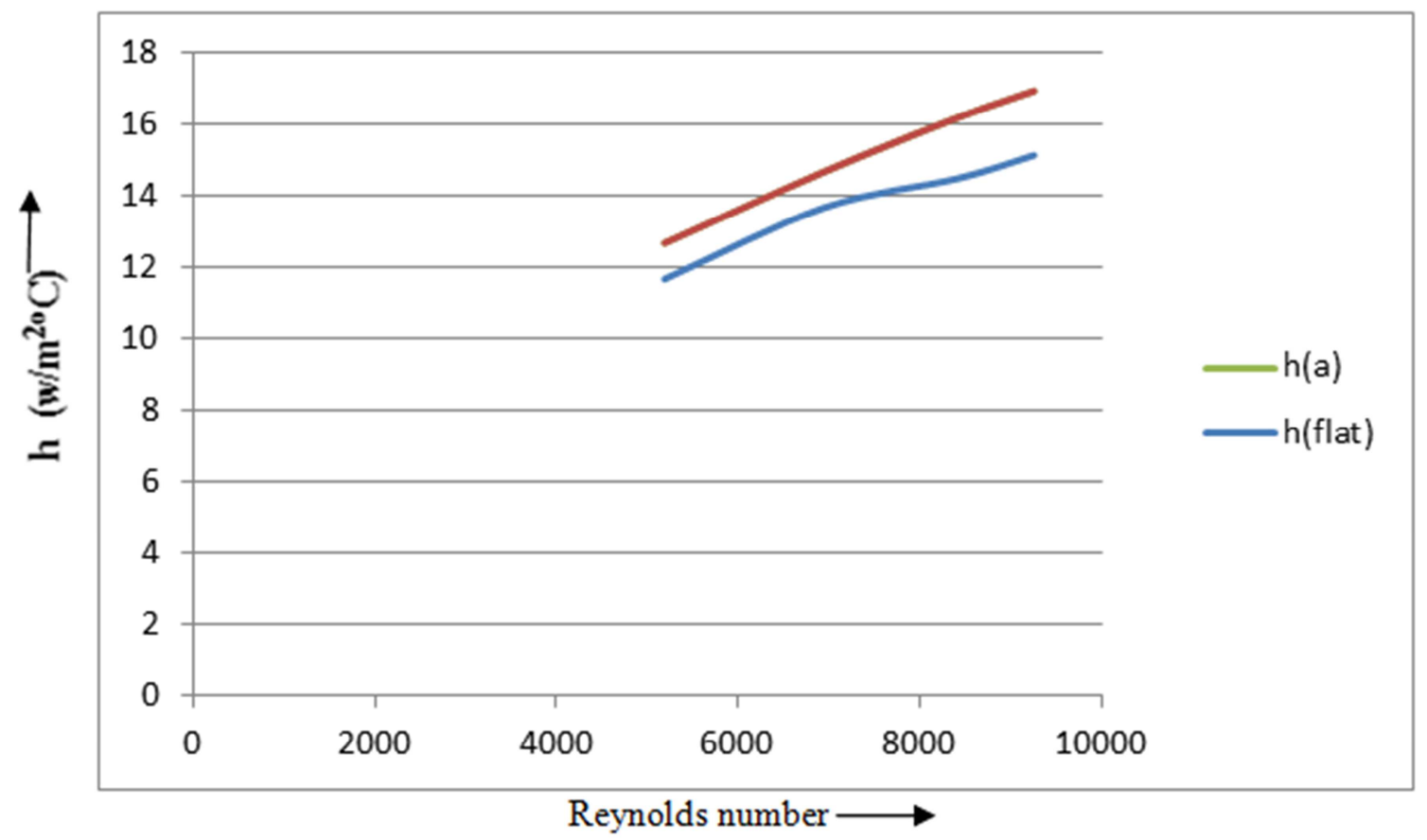

Figure 6. Variation of Heat transfer coefficient with Reynolds number(case.a.). 




Figure 7. Variation of Heat transfer coefficient with Reynolds number(case.b.).

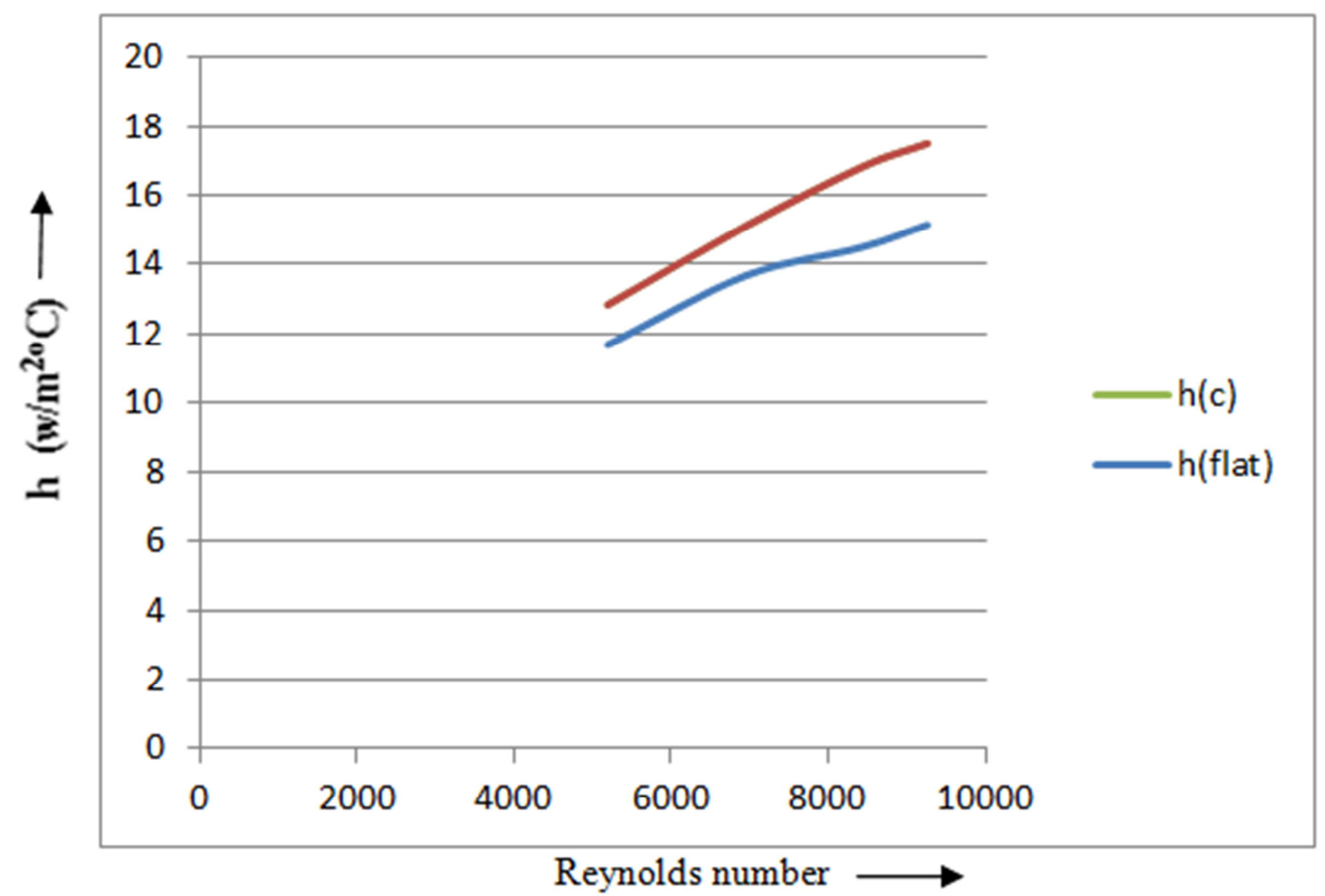

Figure 8. Variation of Heat transfer coefficient with Reynolds number(case.c.).

Figure 6, 7, 8 shows the variation of heat transfer coefficient ' $h$ ' with Reynolds number 'Re' for the various cases considered. It is obvious that ' $h$ ' increases with ' $R e$ ' as expected because the development of the thermal boundary layer is delayed or disrupted \& hence enhances the local heat transfer in the reattachment region and wake region and increases the heat transfer coefficient [3]. 




Figure 9. Variation of Heat transfer rate with Reynolds number(case.a.).

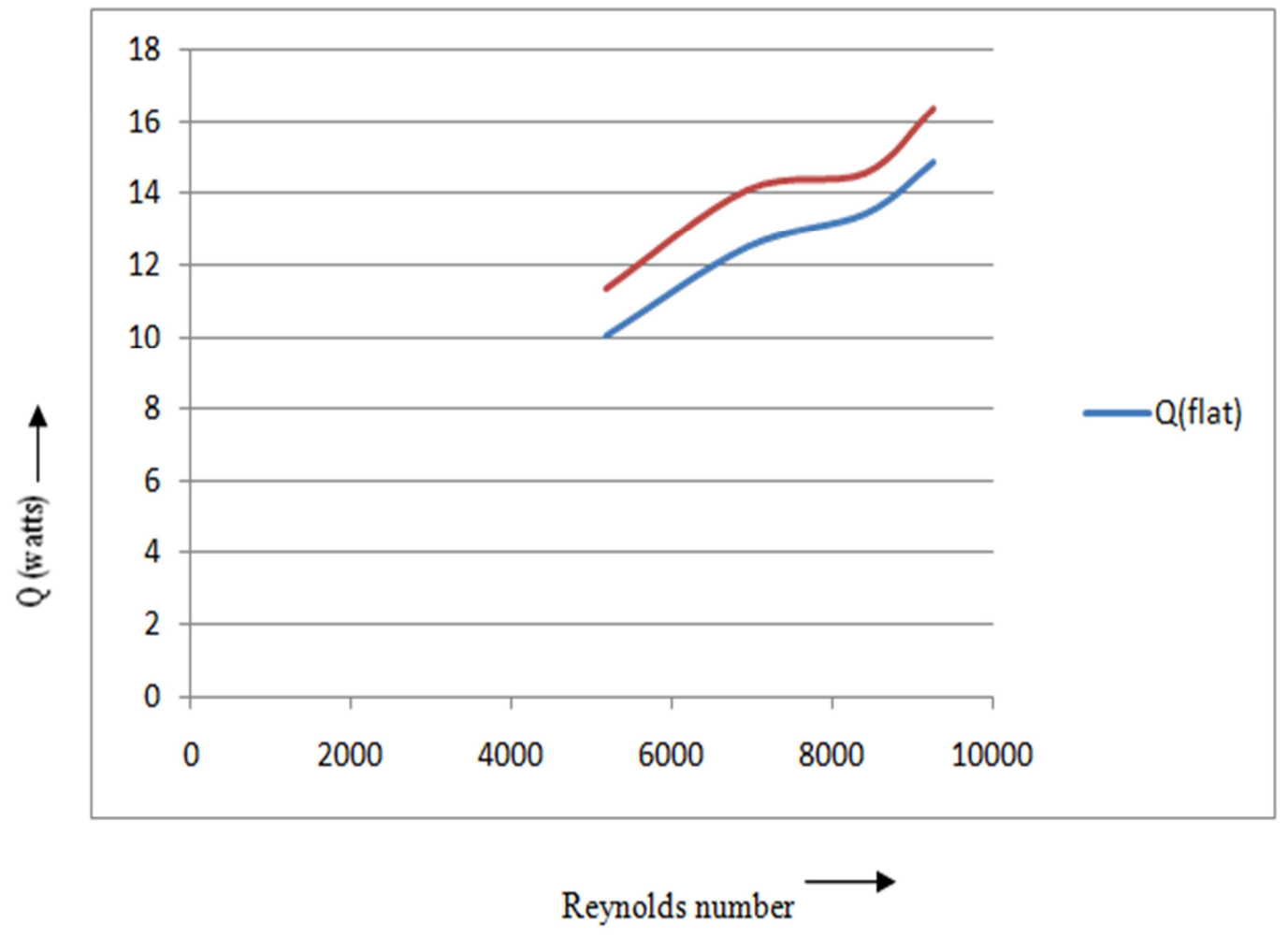

Figure 10. Variation of Heat transfer rate with Reynolds number(case.b.). 


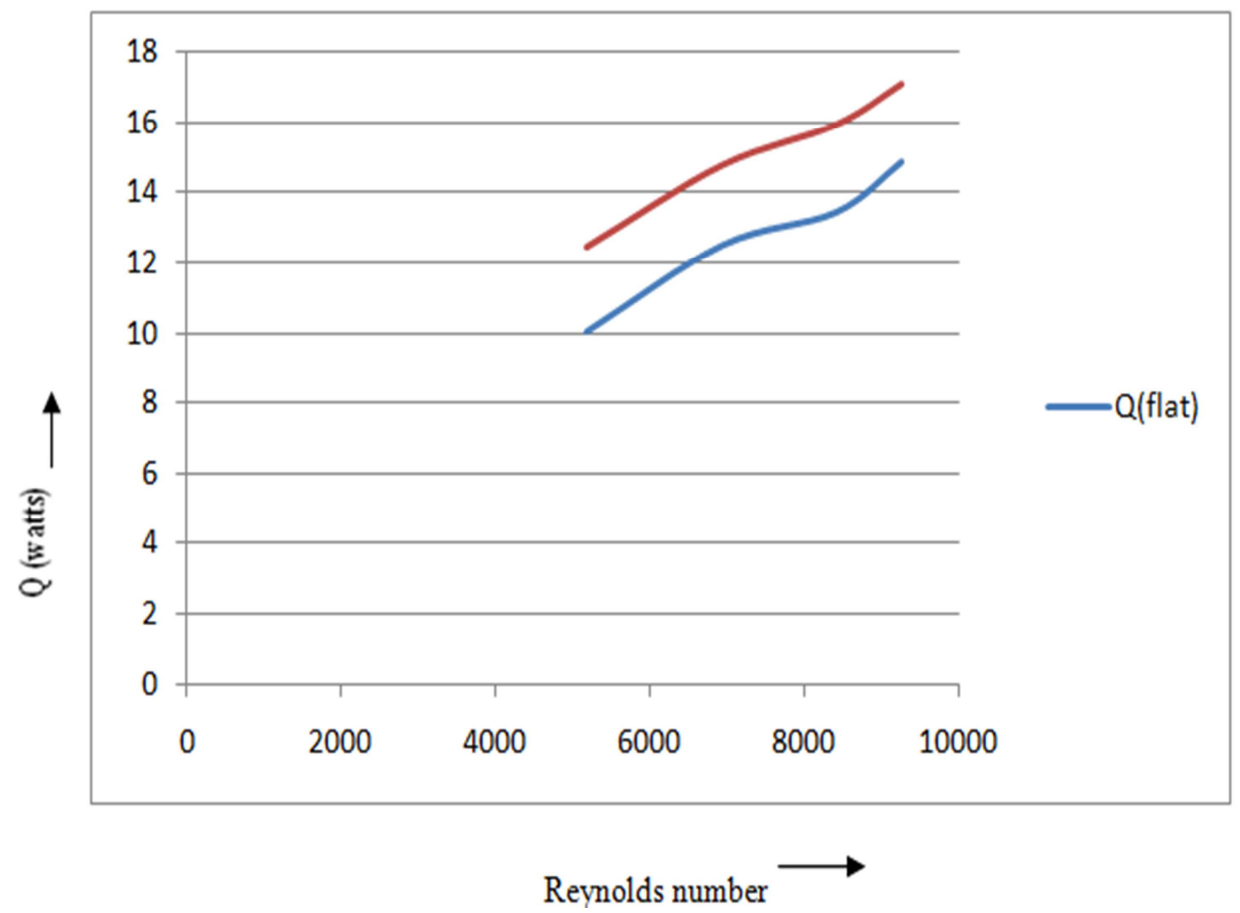

Figure 11. Variation of Heat transfer rate with Reynolds number(case.c.).

Figure 9, 10, 11 shows variation of Heat transfer rate 'Q' with Reynolds number ' $R e$ ' for the various cases considered. It can be seen that again ' $Q$ ' increases as 'Re' increases in all the three cases. It can also be seen that ' $Q$ ' is very much higher for case c(dimples diameter decreasing centrally) because of increased flow area as compared to the other two cases. It can also be seen that for high Reynolds number the ' $\mathrm{Q}$ ' curve for case $\mathrm{c}$ is higher than the curve of case $\mathrm{b} \&$ case c. Hence it can be concluded that case c helps in better enhancing the heat transfer compared to case $a \&$ case $b$.

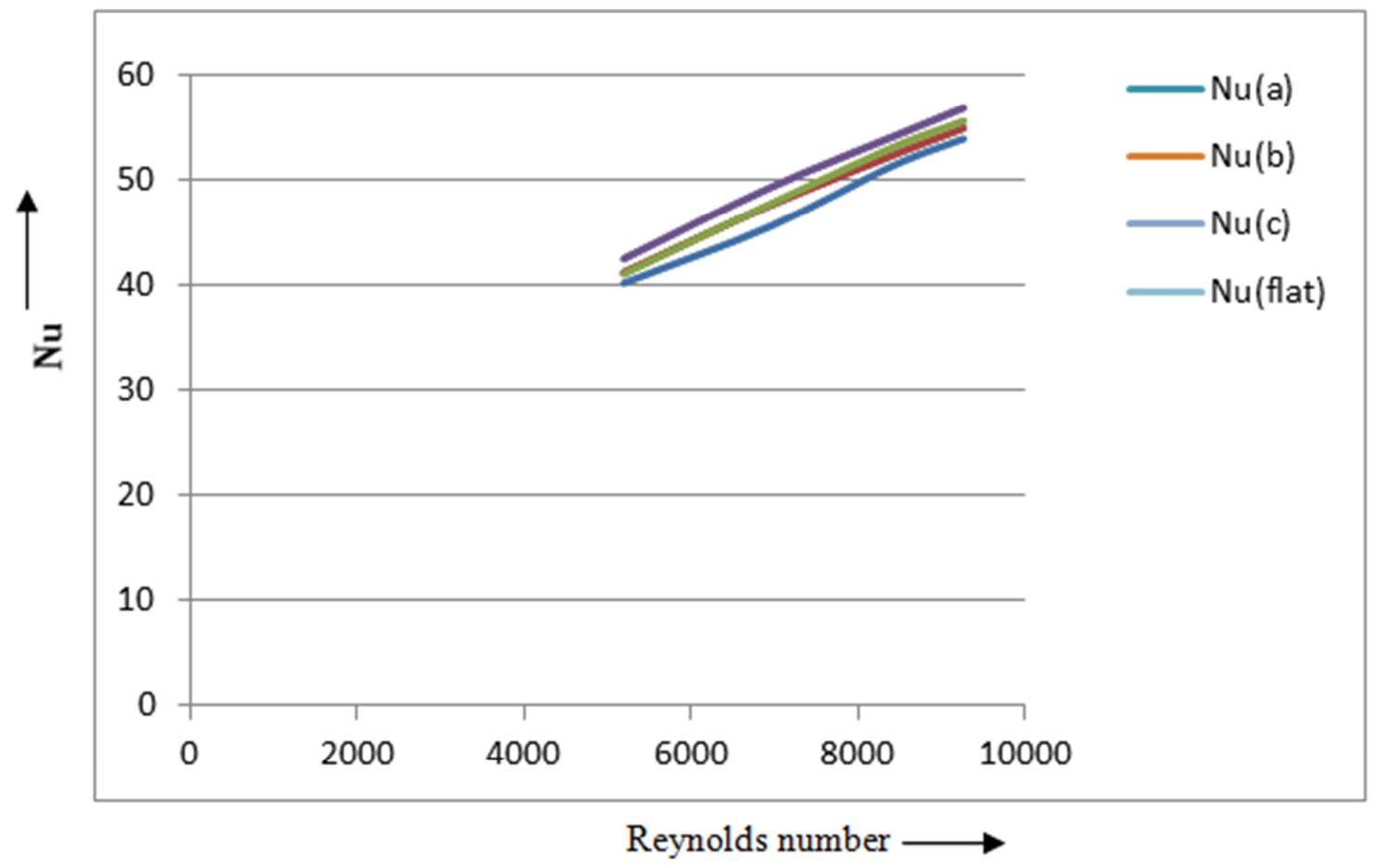

Figure 12. Variation of Nusselt number with Reynolds number.

Figure 12 shows comparison of Nusselt number ' $\mathrm{Nu}$ ' with Reynolds number 'Re' for the all the three cases considered. It can be seen that ' $\mathrm{Nu}$ ' increases as 'Re' increases in all the three cases. It can also be seen that the variation for the first two cases is very less compared to the third case may be due the fact that the dimple diameter is not increased or decreased 
centrally where the pronounce effect of heat transfer will occur so the variation is negligibly small.

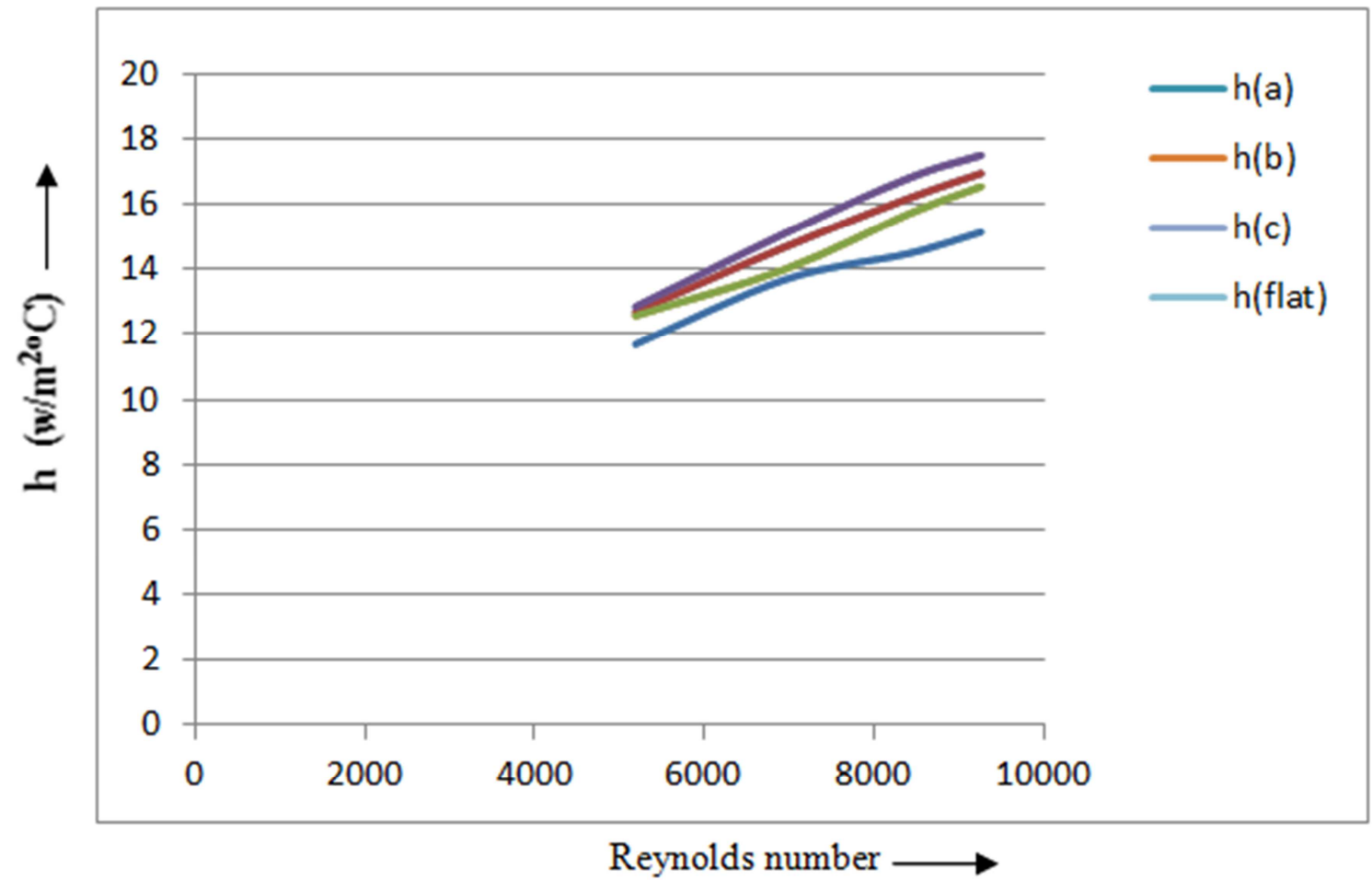

Figure 13. Variation of Heat transfer coefficient with Reynolds number.

Figure 13 shows the comparison of the variation of heat transfer coefficient ' $h$ ' with Reynolds number 'Re' for the various cases considered. It is obvious that ' $h$ ' increases with 'Re' as expected and it is also observed that heat transfer coefficient is high for the third case (case of dimple diameter decreasing centrally) due to higher heat transfer rate occurring at the central region where the fluid flow rate is highest compared to other two cases.

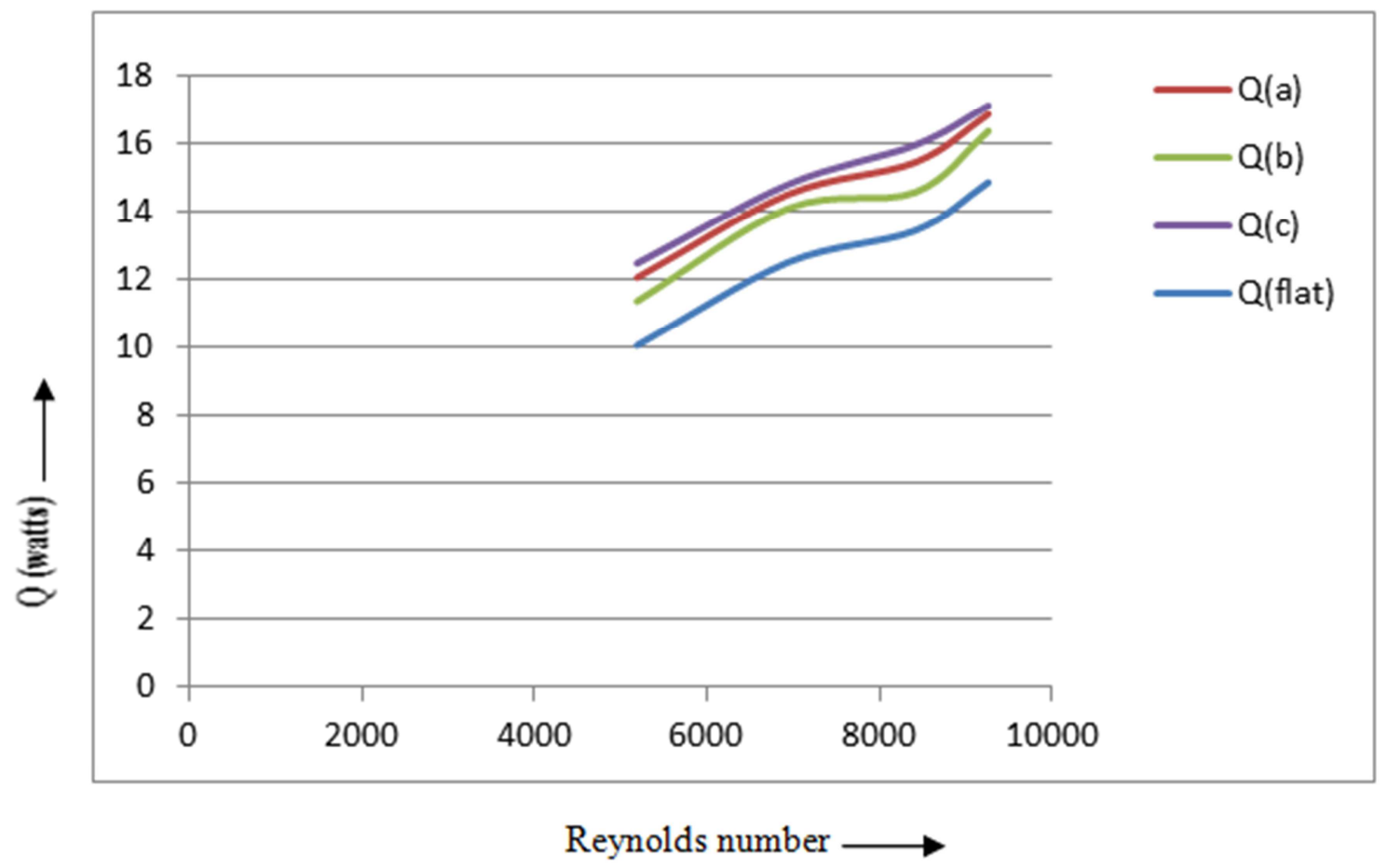

Figure 14. Variation of Heat transfer rate with Reynolds number.

Figure 14 showsthe variation of Heat transfer rate ' $Q$ ' with Reynolds number 'Re' for the various cases considered. It can be seen that again ' $Q$ ' increases as ' $R e$ ' increases in all the three cases. It can also be seen that ' $Q$ ' is very much 
lower for case b \& is highest for case c because of increased flow rate as compared to the other two cases. It can also be seen that for high Reynolds number the ' $Q$ ' curve for case $c$ is higher than the curve of case $b \&$ case $c$. Hence it can be concluded that case $\mathrm{c}$ helps in better enhancing the heat transfer compared to case a $\&$ case b.

\section{Conclusion}

In this experimental work an investigation of the effect of air flow over a flat plate with different diameter dimples on the flat plate is carried out. The main conclusions of the work were:

- Nusselt number increases with Reynolds number for all the three cases of dimple arrangement considered due to direct flow impingement on the downstream boundary and strengthened flow mixing by the vortices at the downstream.

- Case 'c' dimple arrangement has highest Nusselt number because of the strong flow impingement on the upstream side of these dimples. Case a \& b dimple arrangement gives nearly the same value of Nusselt number.

- Heat transfer coefficient increases with Reynolds number for all the three cases of dimples arrangement considered due to the disruption of the thermal boundary layer development $\&$ hence enhance the local heat transfer in the reattachment and wake regions.

- Case 'c' dimple arrangement gives slightly higher value of heat transfer coefficient as compared to case 'a' \& 'b' dimples.

- Case 'c' dimple arrangement has better heat transfer enhancing capacity as compared to case ' $a$ ' \& 'b' because of the increased flow rate in this type ofdimple arrangement. However, the Augmentation depends on the configuration [12].

\section{References}

[1] Dewan, A., Mahanta, P., Raju,K.S., and Kumar.P.S.,Review of passive heat transfer augmentation techniques. Proc. Instn Mech. Engrs, Part A: J. Power and Energy, Vol. 218, pp. 509$527,2004$.

[2] Liu ,S, and Sakr ,M.A., comprehensive review on passive heat transfer enhancements in pipe exchangers. Renewable and Sustainable Energy Reviews, Vol.19 pp. 64-81, 2013.

[3] Zhang, D., Zheng, L., Xie, G., and Xie, Y.,An Experimental Study on Heat Transfer enhancement of Non-Newtonian Fluid in a Rectangular Channel with Dimples/Protrusions, Transactions of the ASME, Vol. 136, pp.021005-10, 2014.
[4] Beves, C.C., Barber, T.J., and Leonardi,E., An Investigation of Flow over Two-Dimensional Circular Cavity. In 15th Australasian Fluid Mechanics Conference, the University of Sydney, Australia, pp.13-17, 2004.

[5] Kuethe A. M., Boundary Layer Control of Flow Separation and Heat Exchange. US Patent No. 1191, 1970.

[6] Afanasyev, V. N., Chudnovsky, Y. P., Leontiev, A. I., and Roganov, P.S., Turbulent flow friction and heat transfer characteristics for spherical cavities on a flat plate. Experimental Thermal Fluid Science, Vol. 7, Issue 1, pp. 1-8, 1993.

[7] Chyu, M. K., Yu, Y., Ding, H., Downs, J. P., and Soechting, F.O., Concavity enhanced heat transfer in an internal cooling passage. In Orlando international Gs Turbine \& Aero engine Congress \& Exhibition, Proceedings of the 1997(ASME paper 97-GT-437), 1997.

[8] Mahmood, G. I., Hill, M. L., Nelson, D. L., Ligrani, P. M., Moon, H.K., and Glezer, B., Local heat transfer and flow structure on and above a dimpled surface in a channel. $\mathrm{J}$ Turbomach, Vol.123, Issue 1, pp: 115-23, 2001.

[9] Yonghui Xie, Huancheng Qu ,Di Zhang ,Numerical investigation of flow and heat transfer in rectangular channel with teardrop dimple/protrusion. International Journal of Heat and Mass Transfer, Vol 84 pp.486-496,2015

[10] Farhad sangtarash \& hosseinshokuhmand ,Experimental and numerical investigation of the heat transfer augmentation and pressure drop in simple, dimpled and perforated dimpled louver fin banks with an in-line or staggered arrangement ,Applied Thermal Engineering Vol 82 ,pp194205, 2015.

[11] Gadhave, G., and Kumar. P., Enhancement of forced Convection Heat Transfer over Dimple Surface-Review. International Multidisciplinary e - Journal .Vol-1, Issue-2, pp.51-57, 2012

[12] Katkhaw, N., Vorayos, N., Kiatsiriroat, T., Khunatorn, Y., Bunturat, D., and Nuntaphan., A. Heat transfer behavior of flat plate having 450 ellipsoidal dimpled surfaces. Case Studies in Thermal Engineering, vol.2, pp. 67-74, 2014.

[13] Patel,I.H ., and Borse ,S.H. Experimental investigation of heat transfer enhancement over the dimpled surface. International Journal of Engineering Science and Technology, Vol.4, Issue 6, pp.3666-3672, 2012.

[14] Faheem Akhtar, Abdul Razak R Kaladgi and Mohammed Samee, Heat transfer augmentation using dimples in forced convection -an experimental approach. Int. J. Mech. Eng. \& Rob. Res. Vol 4,Issue 1 ,pp 150-153,2015

[15] Faheem Akhtar, Abdul Razak R Kaladgi and Mohammed Samee, Heat transfer enhancement using dimple surfaces under natural convection-an experimental study, Int. J. Mech. Eng. \& Rob. Res. Vol 4,Issue 1 ,pp 173-175,2015 